\title{
Two-Handed Marking Menus for Multitouch Devices
}

\author{
KENRICK KIN, University of California, Berkeley and Pixar Animation Studios \\ BJÖRN HARTMANN and MANEESH AGRAWALA, University of California, Berkeley
}

\begin{abstract}
We investigate multistroke marking menus for multitouch devices and we show that using two hands can improve performance. We present two new two-handed multistroke marking menu variants in which users either draw strokes with both hands simultaneously or alternate strokes between hands. In a pair of studies we find that using two hands simultaneously is faster than using a single, dominant-handed marking menu by $10-15 \%$. Alternating strokes between hands doubles the number of accessible menu items for the same number of strokes, and is similar in performance to using a one-handed marking menu. We also examine how stroke direction affects performance. When using thumbs on an iPod Touch, drawing strokes upwards and inwards is faster than other directions. For two-handed simultaneous menus, stroke pairs that are bilaterally symmetric or share the same direction are fastest. We conclude with design guidelines and sample applications to aid multitouch application developers interested in using one- and two-handed marking menus.
\end{abstract}

Categories and Subject Descriptors: H.5.2 [Information Interfaces and Presentation]: User Interfacesinteraction styles

General Terms: Design, Human Factors

Additional Key Words and Phrases: Two-handed multistroke marking menus, multitouch

ACM Reference Format:

Kin, K., Hartmann, B., and Agrawala, M. 2011. Two-handed marking menus for multitouch devices. ACM Trans. Comput.-Hum. Interact. 18, 3, Article 16 (July 2011), 23 pages.

DOI $=10.1145 / 1993060.1993066$ http://doi.acm.org/10.1145/1993060.1993066

\section{INTRODUCTION}

Marking menus are gesture-based menus that allow users to select a menu item by drawing a directional stroke [Kurtenbach 1993]. Multistroke marking menus extend the basic technique and allow users to efficiently traverse a hierarchy of submenus by drawing a sequence of strokes [Zhao and Balakrishnan 2004]. These techniques are effective because they are simple to perform. Strokes are scale-independent and users can draw them in-place and in an eyes-free manner. Extensive studies have shown that users can draw directional strokes quickly and accurately [Kurtenbach and Buxton 1993, 1994; Moyle and Cockburn 2002; Zhao et al. 2006]. Marking menus also facilitate novice to expert transition. However, marking menu research has primarily focused on studying the use of one-handed marking menus with either mouse or stylus-based input devices.

Multitouch input devices have recently become a popular alternative to both the mouse and stylus, particularly for small-screen personal devices such as the Apple

Authors' address: K. Kin (corresponding author), University of California, Berkeley and Pixar Animation Studios; email: kenrick.kin@gmail.com.

Permission to make digital or hard copies of part or all of this work for personal or classroom use is granted without fee provided that copies are not made or distributed for profit or commercial advantage and that copies show this notice on the first page or initial screen of a display along with the full citation. Copyrights for components of this work owned by others than ACM must be honored. Abstracting with credit is permitted. To copy otherwise, to republish, to post on servers, to redistribute to lists, or to use any component of this work in other works requires prior specific permission and/or a fee. Permissions may be requested from Publications Dept., ACM, Inc., 2 Penn Plaza, Suite 701, New York, NY 10121-0701 USA, fax +1 (212) 869-0481, or permissions@acm.org.

(c) 2011 ACM 1073-0516/2011/07-ART16 $\$ 10.00$

DOI 10.1145/1993060.1993066 http://doi.acm.org/10.1145/1993060.1993066 


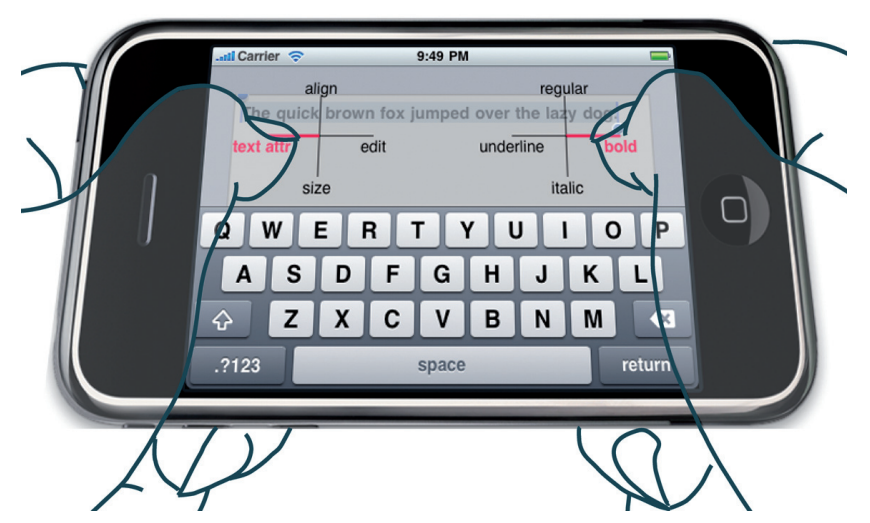

Fig. 1. Using a two-handed ordered marking menu the left thumb strokes to select "Text Attributes" and then the right thumb selects "Bold" to modify the sentence. With a two-handed simultaneous marking menu, users draw both strokes at the same time.

iPhone/iPod Touch [Apple] and for large-screen co-located collaborative work surfaces [MERL; Microsoft; PerceptivePixel]. Marking menus are a good match for small devices because they require very little screen space to perform. More generally, because marking menus are gesture-based techniques, they do not require precise targeting and thereby circumvent the fat finger problem [Potter et al. 1988]. Unlike a mouse or stylus, multitouch devices detect multiple points of contact and therefore support two-handed interactions. These devices have the potential to significantly increase the efficiency of interaction because users can overlap their hand motions and work with both hands in parallel.

In this article, we examine the speed and accuracy of one and two-handed multistroke marking menus with two multitouch devices: a small-screen Apple iPod Touch operated with the thumbs (Figure 1) and a Fingerworks iGesture [Fingerworks] operated with the index or middle fingers as one would on a large-screen interactive surface. Our primary contribution is the design and evaluation of two new two-handed variants of multistroke marking menus.

Two-Handed Simultaneous. Users draw two strokes, one with each hand, at the same time. This variant is designed to maximize parallelism in hand motions and thereby offer the fastest selection times.

Two-Handed Ordered. Users alternate the hand used to draw each stroke. Since either hand (left or right) can start the stroke sequence, this variant offers access to twice as many menu items for the same number of strokes, while also allowing for some temporal overlap in hand motions.

We compare the speed and accuracy of these two-handed designs to one-handed marking menus, We find that the two-handed simultaneous technique outperforms the single, dominant-handed technique by $10-15 \%$ in total time. However, we show through a longitudinal study spanning four hours over five days, that obtaining this performance gain requires some practice. As users gain familiarity with the two-handed design, selecting a menu item becomes proceduralized and autonomous, allowing two hands to outperform one hand. While the two-handed ordered approach is not significantly faster than the one-handed approach in total time, it doubles the number of accessible menu items.

The anatomy of the hand imposes constraints on the range of motions different fingers can make. It may be easier to draw individual strokes or pairs of strokes in some 
directions rather than others. To better understand these constraints, we also examine how stroke direction affects speed and accuracy. In the context of one-handed marking menus, we find that drawing a stroke using either thumb is fastest when drawing upwards or inwards with respect to the hands compared to the other directions. In the context of two-handed simultaneous marking menus, we find that drawing pairs of strokes in which the left and right strokes are either bilaterally symmetric or share the same direction is faster than drawing the other pairs. We conclude with a set of design guidelines that multitouch designers should consider when developing one- or twohanded multistroke marking menus at both handheld (iPhone/iPod Touch) and larger (iGesture/iPad) scales. We present several demonstration applications that show how two-handed marking menus could be used to support real-world tasks and to facilitate the transition from novice to expert use.

\section{RELATED WORK}

Our two-handed multistroke marking menus build on three areas of related work.

Hierarchical Marking Menus. Kurtenbach [1993] and Kurtenbach and Buxton [1993, 1994] introduced marking menus and showed that these menus exhibit a number of desirable properties. Marking menus are scale-independent-the selection depends only on the orientation of the stroke, not on its length, and therefore users can efficiently draw short strokes with ballistic motions to select items [McGuffin et al. 2002]. Users can draw strokes in-place and do not have to make large round-trip traversals to select items from a fixed location menu. Moreover, users can draw the straight-line strokes in an eyes-free manner without diverting attention from their primary task. Finally, marking menus provide a seamless novice-to-expert transition path; novices draw exactly the same selection strokes as experts.

However, a drawback of marking menus is that selection accuracy depends on menu breadth, or the number of items that appear in the menu. Kurtenbach and Buxton [1993] found that accuracy declines substantially when breadth is greater than eight items. Compound-stroke [Kurtenbach and Buxton 1993] and multistroke [Zhao and Balakrishnan 2004] marking menus allow for hierarchical traversal of marking menus using either zig-zag strokes or a sequence of strokes. At breadth-8, however, these techniques perform well only up to depth-2 or depth-3 respectively. More recent techniques have used additional stroke attributes such as stroke position [Zhao et al. 2006] and curvature [Bailly et al. 2008] to further increase menu breadth. All of these techniques have focused on one-handed input. While we adopt the multistroke approach, our work focuses on the design of two-handed marking menus that increase either the selection speed or the number of accessible menu items.

Marking Menus on Touch Devices. Touchpads that can track a single point of contact have been commonplace on laptops for the last decade. Balakrishnan and Patel [1998] integrated such a touchpad with a mouse to allow the nondominant hand to select commands using a compound-stroke marking menu. Isokoski and Käki [2002] found that curved strokes were more accurate but slower in movement time than drawing straight-line selection strokes. Touch-sensing screens are now commonplace on mobile devices. Karlson et al. [2005] used directional strokes for thumb-based navigation on a PDA. Yatani et al. [2008] used a combination of position and directional strokes to disambiguate the selection of closely packed items on a touch-based mobile device. Lepinski et al. [2010] developed chording marking menus in which users draw simple directional strokes using combinations of fingers on a single hand. While all of these stroke-based techniques are designed for touch-based devices, none of them have examined the use of multiple strokes in different directions or two-handed interactions. 
Bimanual Interaction Techniques. The Kinematic Chain Theory of Guiard [1987] details the way hands work together to perform tasks in parallel. Many bimanual interaction techniques assign asymmetric roles to the hands where the nondominant hand sets the frame of reference and the dominant hand performs fine-scale interactions within this reference frame [Balakrishnan and Hinckley 1999; Buxton and Myers 1986; Hinckley et al. 1997; Kabbash et al. 1994]. Other techniques assign symmetric roles in which both hands perform similar actions [Balakrishnan and Hinckley 2000; Casalta et al. 1999; Latulipe et al. 2005, 2006; Owen et al. 2005].

Odell et al. [2004] presented an asymmetric bimanual marking menu technique in the context of a shape drawing system. The dominant hand selects a shape and the nondominant hand selects a command to perform on this shape using a marking menu. Unlike this approach, we develop symmetric two-handed marking menus in which both hands perform the same actions. By splitting the strokes of a multistroke marking across both hands, we allow for overlap in the hand motions and increase the speed of the interaction.

Controllers for console-based gaming systems such as the Xbox [Xbox] usually include two joysticks, one for each hand. Wilson and Agrawala [2006] developed a two-joystick based text-entry system using an onscreen keyboard and showed that such a symmetric bimanual approach is faster than using the default, single joystick technique. TwoStick [Költringer et al. 2007] extends Quikwriting [Perlin 1998], a technique that uses directional joystick movements to enter text, for use with two joysticks. Weegie [Weegie] is another two-stick-based text entry system in which each stick operates a separate marking menu. Unlike our two-handed marking menus, the two menus in Weegie work independently of one another.

\section{DESIGNING TWO-HANDED MARKING MENUS}

One-handed multistroke marking menus have proven to be effective for use with a stylus or mouse because they are scale-independent, in-place, eyes-free, and provide a seamless novice-to-expert transition. We extend multistroke marking menus for use on multitouch devices by splitting the stroke sequence between two hands. We consider several aspects of two-handed operation that can further increase menu selection performance.

Temporal Overlap of Motion. Users can temporally overlap motions of their hands and this parallelism can reduce the time required to complete the interaction.

Hand Identity (Left/Right). Multitouch devices detect multiple points of contact; we can use heuristics based on contact position to infer the hand corresponding to each contact-for example, the leftmost touch is from the left hand and the rightmost touch is from the right hand. We can then increase the number of menu items that are accessible with a single stroke, by assigning a different set of items to each hand. With a hierarchical marking menu of depth- $N$ in which either hand can draw each stroke in the sequence, we can use hand identity to increase the number of accessible items by a factor of $2^{N}$. However, if the same hand is used to draw more than one consecutive stroke the potential for temporal overlap in the hand motions is reduced.

Chunking. Buxton [1986] has shown that users can mentally group together frequently co-occurring compound motor tasks into a single chunk that they automatically perform together. With two-handed multitouch devices, users can draw a pair of strokes simultaneously, one with each hand, and may learn to chunk these pairs together into a single action. Thus, users can mentally flatten two levels of a multistroke hierarchy into a single level, and convert a breadth- $M$, depth-2 marking menu into a breadth- $M^{2}$, depth-1 menu. Such increased breadth may allow interface designers and users to fit more items in a single cognitive grouping. 
Based on these design considerations, we propose the following two-handed multistroke marking menus designs:

\subsection{Two-Handed Simultaneous Marking Menus (2HS)}

Users simultaneously draw two strokes, one with each hand. Users can draw additional stroke pairs to traverse a menu hierarchy. This variant is designed to maximize the temporal overlap of motions and also facilitate chunking of the stroke pairs into a single action. However, this variant does not use hand identity as an extra bit of information. Therefore, for a given number of strokes, it does not increase the number of accessible menu items over the one-handed multistroke marking menu design. However, when users chunk pairs of simultaneous strokes, this variant can be considered as flattening the depth and thus, squaring the breadth of the menu.

\subsection{Two-Handed Ordered Marking Menus (2HO)}

Users draw a sequence of strokes with alternating hands. Although the strokes must be drawn in order, the ordering only depends on the start-time of each stroke. Users can begin a second stroke before the first stroke is complete to increase temporal motion overlap. This variant considers hand identity, but because the hands are forced to alternate, only the hand used to initiate the stroke sequence can vary (left or right), providing one additional bit of information. Thus, this approach doubles the number of accessible menu items accessible for a fixed number of strokes. Although using hand identity could in theory increase the number of accessible menu items by a factor of $2^{N}$, because our ordered design forces users to alternate hands, it trades off access to fewer menu items for an increase in the potential for temporal motion overlap in drawing the strokes.

\section{USER STUDY 1}

To investigate the performance benefits of our two-handed multistroke marking menu designs we conducted a first user study comparing both of our designs to standard one-handed multistroke marking menu designs. To simplify analysis we selected only right-hand dominant participants, but we included both right- and left-handed unimanual marking menus in our study. We conducted the experiment using two multitouch devices - an iPod Touch to represent handheld interaction and the iGesture to represent larger-screen interaction. Our hypotheses are the following.

$H 1$. The two-handed simultaneous menu will be faster for selecting menu items than all other menus including two-handed ordered and one-handed menus. The two-handed simultaneous design maximizes the opportunity to temporally overlap hand motions and should therefore reduce selection time.

H2. The two-handed ordered menu will be faster than a one-handed marking menu for selecting a menu item. Users do not have to wait for one stroke to finish before starting the next stroke and may be able to overlap their hand movements.

H3. Of the one-handed conditions the right-handed multistroke marking menu will outperform the left-handed multistroke marking menu. Since our participants are right-handed, their dominant hand should move more quickly and accurately than their nondominant, left hand.

Two-handed motions on touch devices may have different constraints than the mouse and stylus strokes investigated in prior work [Kurtenbach and Buxton 1993, 1994; Moyle and Cockburn 2002; Zhao et al. 2006; Zhao and Balakrishnan 2004]. Studies of bimanual motion suggest that mirrored pairs of strokes may be easier to draw than other pairs [Kelso 1984; Mechsner et al. 2001]. Thus, our study also investigates how 


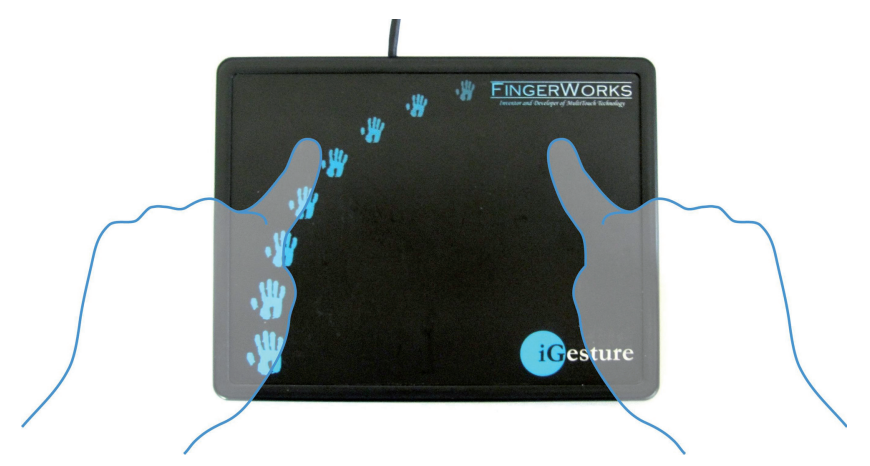

Fig. 2. The Fingerworks iGesture multitouch pad.

well users can draw directional strokes with their left and right hands individually, and how well they can draw pairs of strokes with two hands.

\subsection{Participants and Apparatus}

We recruited 16 right-handed participants (12 male, 4 female, between 21 and 26 years old). All were experienced computer users and ten were experienced iPhone or iPod Touch users. None of the participants had experience with marking menus or with large multitouch screens. Participants performed the experiment on two multitouch devices.

Apple iPod Touch. The 2nd generation iPod Touch is a commonly used handheld device with a multitouch screen. It has a working area of $7.5 \times 5 \mathrm{~cm}$ and display resolution of $480 \times 320$ pixels. Participants used their thumbs on this device.

Fingerworks iGesture. The iGesture is an indirect multitouch pad with a working area of $16.5 \times 12.4 \mathrm{~cm}$ (Figure 2) mapped by absolute coordinates to a $40.6 \times 30.5 \mathrm{~cm}$ Dell monitor with a display resolution of $1600 \times 1200$ pixels. The study ran on Mac OS $\mathrm{X}$. Participants used either their index fingers or middle fingers on this device.

\subsection{Task and Stimuli}

We designed the study to test expert-level performance. However, our participants had practically no prior experience using marking menus. Training participants to use multistroke marking menus with realistic menu items would force them to learn a complex menu organization. But, an expert user would require little effort to recall the necessary strokes for a command. To better elicit expert-level performance with far less training, we adopted the strategy of previous marking menu studies [Kurtenbach and Buxton 1993; Zhao et al. 2006; Zhao and Balakrishnan 2004] and gave participants stimuli in the form of arrows that directly indicated the strokes they should draw.

Examples of the stimuli we used are shown in Figure 3. Arrows appeared in separate columns to indicate the hand that should draw the stroke. Pairs of arrows in the same row indicated strokes that must be drawn simultaneously. Participants had to draw the strokes in order from top to bottom. In the one-handed conditions (not shown in figure), the stimuli only contained arrows in either the left or right column.

To begin a trial, the participant tapped the device with one finger for the one-handed conditions or one finger on each hand for the two-handed conditions. The stimulus appeared at the top of the screen and, following the approach of Zhao et al. [2006], as soon as the participant touched the input device the cue disappeared so that the subject could not read the arrows while drawing marks. This approach was designed to better elicit expert-level performance because it prevented subjects from interleaving 


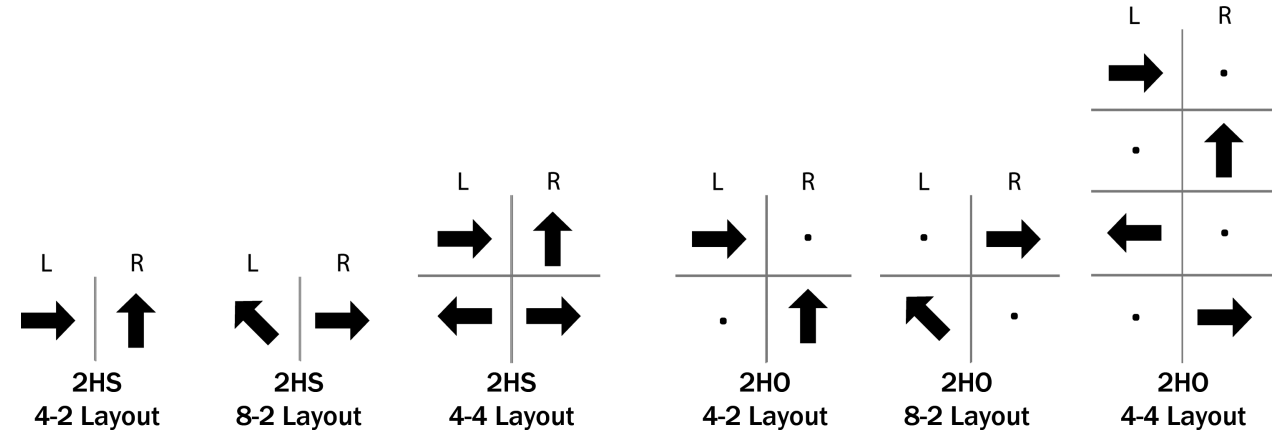

Fig. 3. Example stimuli for the two-handed simultaneous (2HS) and two-handed ordered (2HO) conditions. Arrows appear in separate columns to indicate the hand that should draw the stroke. Pairs of arrows in the same row indicate strokes that must be drawn simultaneously. Participants must draw strokes in order from top to bottom. In the one-handed conditions (not shown), the stimuli only contain arrows in either the left or right column.

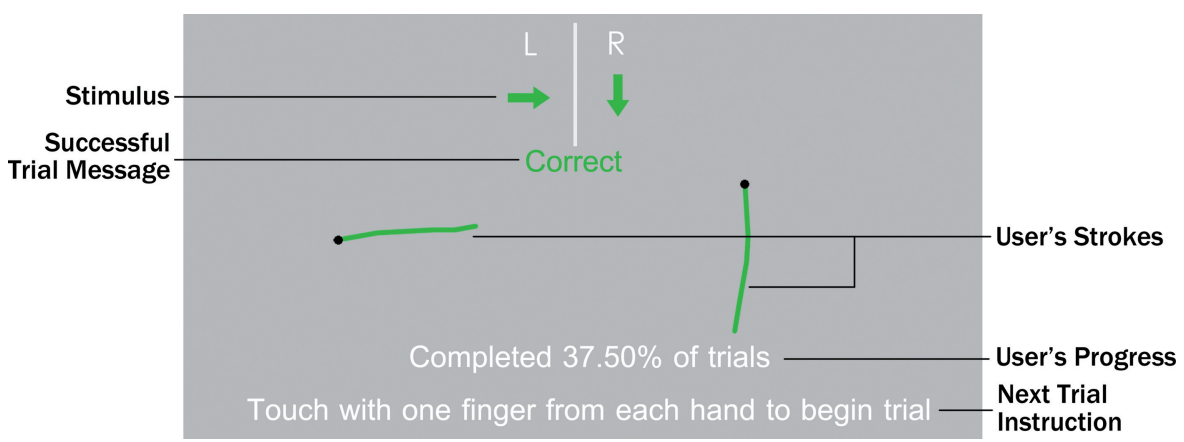

Fig. 4. Screenshot of experimental setup with feedback given after a successful trial.

drawing the strokes and reading the stimulus. We asked the participant to draw the strokes as quickly and accurately as possible.

After the participant made the designated number of strokes (2 or 4 strokes in our experiment), a feedback screen showed whether or not the trial was successful and the percentage of trials completed so far (Figure 4). On correct trials, we colored the strokes and stimuli green, and on incorrect trials we colored them red. The participant could rest between trials while the feedback was onscreen and they could continue to the next trial by tapping on the device.

\subsection{Study Design}

Our experiment used a within subjects design and included three independent variables: device, menu technique, and menu layout. We fully counterbalanced the device variable so that half the participants used the iPod Touch first and the other half used the iGesture first.

Participants had to select menu items using one of four menu techniques; left-handed multistroke marking menu (1HL), right-handed multistroke marking menu (1HR), two-handed simultaneous multistroke marking menu (2HS), or two-handed ordered multistroke marking menu $(2 \mathrm{HO})$. We used a Latin square to counterbalance the ordering of the menu techniques.

For each menu technique we tested three different breadth-depth menu layouts: 4-2, 8-2, and 4-4. Breadth denotes the number of possible directions for each stroke, 
while depth denotes the total number of strokes users must draw. A breadth-4 layout includes only the four cardinal directions, whereas a breadth-8 layout includes both the cardinal and diagonal directions. We only considered the number of strokes in multiples of two, so that strokes could be evenly distributed between hands for the two-handed conditions. Although the two-handed techniques would work with an odd number of strokes, we believe it should be possible to extrapolate performance for those conditions using the data we collected for even numbers of strokes.

We fixed the ordering of the three layouts from least complex to most complex (4-2, 8-2, 4-4). As the number of accessible menu items or stroke combinations increases, more trials are necessary to obtain good coverage. Our three layouts allow a total of 16 , 64 , and 256 possible stroke combinations and we used 24,32 , and 32 trials respectively. For the 4-2 layout, each stroke combination was performed at least once, in randomized order. For the 8-2 layout, there are four possible pairs of on- and off-axis strokes: on-on, on-off, off-on, off-off. We randomized the stroke combinations such that each participant performed 8 trials from each axis grouping. For the 4-4 layout, we randomly chose the stroke combination from amongst all possible combinations for that layout. For the two-handed ordered condition, we randomized the order of the starting hand with half the trials beginning with the left hand.

We considered a trial to be a miss if any one of the strokes was drawn in an incorrect direction. To check for misses, we compared the angle of the line segment connecting the start and end points of the drawn stroke with the angle of each possible stroke direction in the menu. If the angle of the drawn stroke was closest to the angle cued in the stimulus it was considered correct; otherwise, it was considered a miss. We added each missed trial to the end of the trial queue so that users would have to perform it again until successful.

Before testing each menu layout, we gave participants a practice block to train them in reading the stimulus and move them towards expert-level performance. For the 4-2 conditions, we required 20 practice trials, while we required eight practice trials for the 8-2 and 4-4 conditions. In all cases participants had the option to continue practicing until they felt comfortable with the task. The entire experiment took each participant roughly one hour.

We measured four dependent variables: reaction time, movement time, total time, and accuracy. Reaction time was the interval between the first display of the stimulus and the start of the touch beginning the first stroke. It represents the time required for participants to process the stimulus and decide which strokes to draw. Movement time was the interval between the first touch and completion of all strokes and represents the time required to physically draw the strokes. Total time was the sum of the reaction and movement times. We only considered timing data from correct trials to better account for expert-level performance. We computed accuracy as the fraction of correctly performed trials to the total number of incorrect trials. We did not include the trials that were added to the end of the queue as the result of a miss in our accuracy measure.

\section{RESULTS}

We performed a 2 (device) $\times 4$ (technique) $\times 3$ (layout) repeated measures ANOVA for each dependent variable. The average total, reaction, and movement times are shown in Figure 5 for the iPod Touch and in Figure 6 for the iGesture. Figures 7 and 8 show the average accuracy rates for the iPod Touch and iGesture respectively.

For each of the menu layouts, we also compared the performance between same-axis stroke pairs and different-axis stroke pairs. Finally, for the two-handed conditions, we examined differences in the amount of temporal overlap of the two hands and the effects of the starting hand on performance for the ordered technique. 


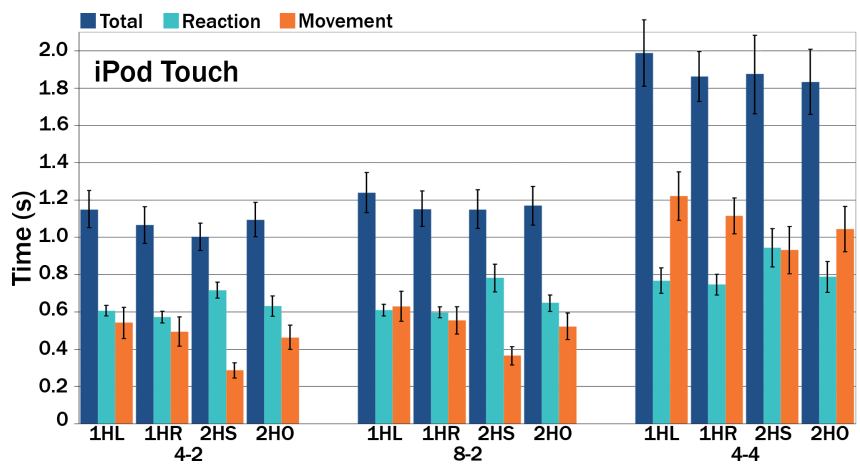

Fig. 5. Average times (with $95 \%$ confidence intervals) for each menu technique and menu layout on the iPod Touch.

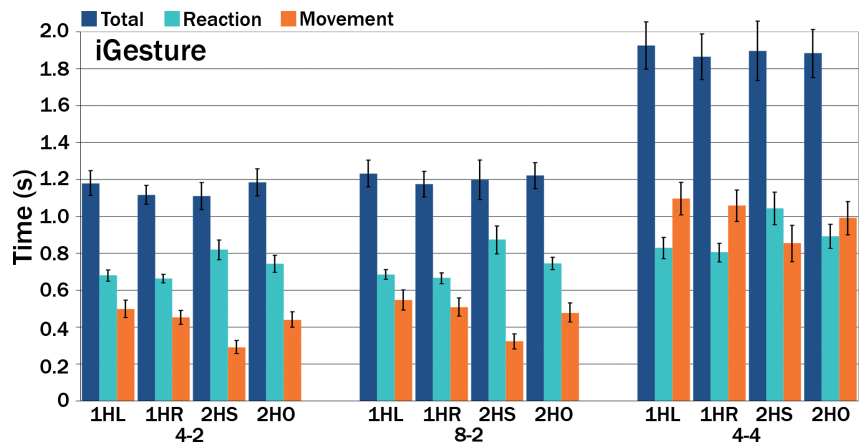

Fig. 6. Average times (with 95\% confidence intervals) for each menu technique and menu layout on the iGesture.

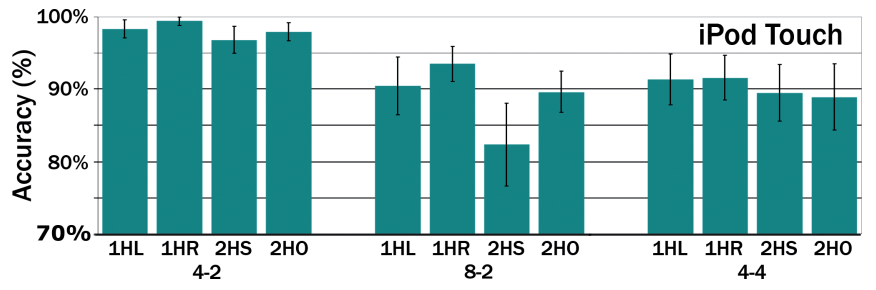

Fig. 7. Average accuracies (with $95 \%$ confidence intervals and baseline value of $70 \%$ ) for each menu technique and menu layout on the iPod Touch.

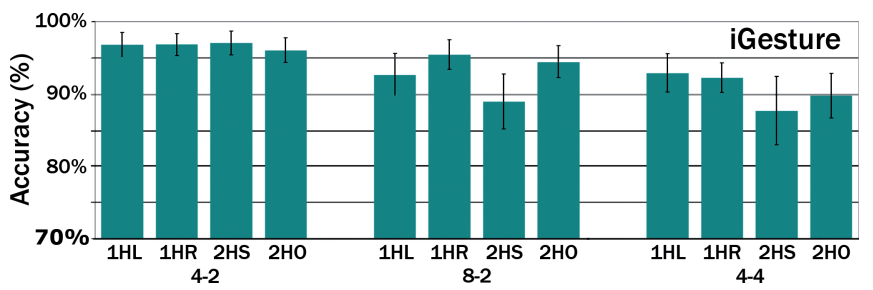

Fig. 8. Average accuracies (with $95 \%$ confidence intervals and baseline value of $70 \%$ ) for each menu technique and menu layout on the iGesture. 
Table I. Average Total Times in Milliseconds and Standard Errors in Parentheses Total time in milliseconds

\begin{tabular}{|c||c|c|c||c|c|c||c|}
\hline \multicolumn{1}{|c||}{} & \multicolumn{3}{c||}{ iPod Touch } & \multicolumn{3}{c||}{ iGesture } & All \\
\cline { 2 - 7 } & $4-2$ & $8-2$ & $4-4$ & $4-2$ & $8-2$ & $4-4$ & $1452(51)$ \\
\hline \hline 1HL & $1149(52)$ & $1238(57)$ & $1988(91)$ & $1179(36)$ & $1231(39)$ & $1926(67)$ & $1372(46)$ \\
1HR & $1065(51)$ & $1151(49)$ & $1861(69)$ & $1116(29)$ & $1174(36)$ & $1864(65)$ & $1372(54)$ \\
2HS & $1003(38)$ & $1149(54)$ & $1875(109)$ & $1110(39)$ & $1197(55)$ & $1897(84)$ & $1372(59)$ \\
2HO & $1094(48)$ & $1170(54)$ & $1832(91)$ & $1183(39)$ & $1221(38)$ & $1884(68)$ & $1397(52)$ \\
\hline
\end{tabular}

Table II. Average Reaction Times in Milliseconds and Standard Errors in Parentheses Reaction time in milliseconds

\begin{tabular}{|c||c|c|c||c|c|c||c|}
\hline \multicolumn{1}{|c||}{} & \multicolumn{3}{c||}{ iPod Touch } & \multicolumn{3}{c||}{ iGesture } & \multirow{2}{*}{ All } \\
\cline { 2 - 7 } & $4-2$ & $8-2$ & $4-4$ & $4-2$ & $8-2$ & $4-4$ & $696(17)$ \\
\hline 1HL & $606(15)$ & $610(17)$ & $767(35)$ & $680(16)$ & $684(14)$ & $829(30)$ & $6965(16)$ \\
1HR & $572(15)$ & $597(14)$ & $747(28)$ & $663(12)$ & $666(15)$ & $805(26)$ & $675(16)$ \\
2HS & $716(24)$ & $783(40)$ & $944(52)$ & $820(28)$ & $874(40)$ & $1042(46)$ & $863(34)$ \\
2HO & $631(29)$ & $648(24)$ & $788(42)$ & $743(25)$ & $744(18)$ & $893(35)$ & $741(24)$ \\
\hline
\end{tabular}

\subsection{Total Time}

As shown in Table I, the average total times pooled across device and layout were $1452 \mathrm{~ms}$ for $1 \mathrm{HL}, 1372 \mathrm{~ms}$ for $1 \mathrm{HR}, 1372 \mathrm{~ms}$ for $2 \mathrm{HS}$, and $1397 \mathrm{~ms}$ for $2 \mathrm{HO}$. We found significant main effects for technique $\left(F_{3,45}=6.45, p=.001\right)$ and layout $\left(F_{2,30}=\right.$ $378.70, p<.001)$. We also found a significant two-way interaction between device and technique $\left(F_{3,45}=5.25, p=.003\right)$ indicating that technique affects total time differently on the two devices. Thus, we performed separate post hoc multiple means comparison tests with Bonferroni correction for each device. For the iPod Touch, we found that $1 \mathrm{HL}$ was significantly slower than the three other techniques across all three layouts $(p<.008)$. For the iGesture, the only significant result we found was that 1HL was slower than 1HR across the three layouts $(p=.006)$.

Results. These results suggest that for both devices and across all layouts, the nondominant, left-handed menu was significantly slower than the dominant, righthanded menu. In addition, the total time required by the two-handed menus was not significantly different from the total time required by the right-handed menu.

\subsection{Reaction Time}

As shown in Table II, the average reaction times pooled across device and layout, were $696 \mathrm{~ms}$ for $1 \mathrm{HL}, 675 \mathrm{~ms}$ for $1 \mathrm{HR}, 863 \mathrm{~ms}$ for $2 \mathrm{HS}$, and $741 \mathrm{~ms}$ for $2 \mathrm{HO}$. We found significant main effects for device $\left(F_{1,15}=88.26, p<.001\right)$; the average reaction time for the iPod Touch $(701 \mathrm{~ms})$ was faster than for the iGesture $(787 \mathrm{~ms})$. We also found significant main effects for technique $\left(F_{3,45}=62.16, p<.001\right)$ and layout $\left(F_{2,30}=61.13\right.$, $p<.001$ ). In addition, we found two-way interactions between technique and device $\left(F_{3,45}=4.36, p=.009\right)$ as well as technique and layout $\left(F_{6,90}=2.57, p=.024\right)$ indicating that technique affects reaction time differently for each device and for each layout. For the iPod Touch, we ran post hoc multiple means comparison tests with Bonferroni correction and found that $2 \mathrm{HS}$ was significantly slower than all other techniques regardless of layout $(p<.003)$. We found only one other significant difference between $1 \mathrm{HR}(597 \mathrm{~ms})$ and $2 \mathrm{HO}(648 \mathrm{~ms})$ for the $8-2$ layout $(p<.020)$. For the iGesture, post hoc multiple means comparison tests with Bonferroni correction found no significant differences between the one-handed techniques (1HL and 1HR) across all three layouts. We also found that $2 \mathrm{HS}$ was significantly slower than all other techniques for every layout $(p<.001)$ with one exception-in the 4-2 layout, we found no significant difference between $2 \mathrm{HS}$ and $2 \mathrm{HO}$. All other pairs were significantly different $(p \leq .026)$. 
Table III. Average Movement Times in Milliseconds and Standard Errors in Parentheses Movement time in milliseconds

\begin{tabular}{|c||c|c|c||c|c|c||c|}
\hline \multicolumn{1}{|c||}{} & \multicolumn{3}{c||}{ iPod Touch } & \multicolumn{3}{c||}{ iGesture } & \multirow{2}{*}{ All } \\
\cline { 2 - 7 } & $4-2$ & $8-2$ & $4-4$ & $4-2$ & $8-2$ & $4-4$ & $756(37)$ \\
\hline \hline 1HL & $542(44)$ & $629(43)$ & $1221(67)$ & $499(26)$ & $547(28)$ & $1097(47)$ & $7597(33)$ \\
1HR & $493(41)$ & $555(39)$ & $1115(51)$ & $453(20)$ & $509(27)$ & $1059(45)$ & $69709(31)$ \\
2HS & $287(21)$ & $366(26)$ & $931(26)$ & $291(18)$ & $324(22)$ & $854(52)$ & $509(37)$ \\
2HO & $463(34)$ & $521(38)$ & $1044(63)$ & $440(24)$ & $477(27)$ & $991(47)$ & $656(35)$ \\
\hline
\end{tabular}

Table IV. Average Accuracy and Standard Errors in Parentheses

\begin{tabular}{|c|c|c|c|c|c|c|c|}
\hline \\
\hline & \multicolumn{3}{|c|}{ iPod Touch } & \multicolumn{3}{|c|}{ iGesture } & \multirow[b]{2}{*}{ All } \\
\hline & $4-2$ & $8-2$ & $4-4$ & 4-2 & 8-2 & $4-4$ & \\
\hline 1HL & $98.4(0.7)$ & $90.4(2.1)$ & $91.4(1.8)$ & $96.8(0.9)$ & $92.7(1.5)$ & $92.9(1.4)$ & $993.7(0.7)$ \\
\hline $1 \mathrm{HR}$ & $99.5(0.4)$ & $93.5(1.3)$ & $91.6(1.6)$ & $96.9(0.8)$ & $95.5(1.1)$ & $92.3(1.1)$ & $94.9(0.5)$ \\
\hline $2 \mathrm{HS}$ & $96.8(1.0)$ & $82.5(2.9)$ & $89.5(2.0)$ & $97.1(0.8)$ & $89.0(2.0)$ & $87.7(2.5)$ & $90.4(1.2)$ \\
\hline $2 \mathrm{HO}$ & $97.9(0.6)$ & $89.6(1.5)$ & $88.9(2.4)$ & $96.1(0.9)$ & $94.5(1.1)$ & $89.9(1.6)$ & $92.8(0.8)$ \\
\hline
\end{tabular}

Results. These results suggest that the two-handed simultaneous technique had the slowest reaction times of all the techniques.

\subsection{Movement Time}

As shown in Table III, the average movement times pooled across device and layout, were $756 \mathrm{~ms}$ for $1 \mathrm{HL}, 697 \mathrm{~ms}$ for $1 \mathrm{HR}, 509 \mathrm{~ms}$ for $2 \mathrm{HS}$, and $656 \mathrm{~ms}$ for $2 \mathrm{HO}$. We found a significant main effect for device $\left(F_{1,15}=7.48, p=.015\right)$; the average movement time for the iGesture $(628 \mathrm{~ms})$ was faster than for the iPod Touch $(681 \mathrm{~ms})$. We also found significant main effects for technique $\left(F_{3,45}=74.91, p<.001\right)$ and layout $\left(F_{2,30}=399.22, p<.001\right)$. Post hoc multiple marginal means comparison tests with Bonferroni correction found that movement times between all pairs of techniques were significantly different ( $p=.035$ for pair $1 \mathrm{HR}-2 \mathrm{HO}, p<.001$ for all other pairs), regardless of device and layout. The only significant two-way interaction was between device and layout $\left(F_{2,30}=5.18, p=.012\right)$.

Results. These results suggest that the two-handed techniques had faster movement times than the one-handed techniques. The two-handed simultaneous technique was faster than the two-handed ordered technique, while the right-handed technique was faster than the left-handed technique.

\subsection{Accuracy}

As shown in Table IV, the average accuracy rates pooled across device and layout, were 93.7\% for $1 \mathrm{HL}, 94.9 \%$ for $1 \mathrm{HR}, 90.4 \%$ for $2 \mathrm{HS}$, and $92.8 \%$ for $2 \mathrm{HO}$. We found significant main effects for technique $\left(F_{3,45}=9.03, p<.001\right)$ and layout $\left(F_{2,30}=32.95, p<.001\right)$. In addition, we found a significant two-way interaction between technique and layout $\left(F_{6,90}=3.36, p=.005\right)$, indicating that technique affects accuracy differently for each layout. Post hoc tests found no significant differences in accuracy between the four techniques for the breadth-4 layouts. However, for the 8-2 layout we did obtain a a significant simple main effect for technique $\left(F_{3,45}=11.63, p<.001\right)$ where $2 \mathrm{HS}$ was less accurate than 1HR $(p<.001)$ and $2 \mathrm{HO}(p=.020)$. We also found a significant two-way interaction between layout and device $\left(F_{2,30}=13.94, p<.001\right)$ as the iPod Touch was more accurate than the iGesture for layout $4-2(p=.012)$, but less accurate for layout 8-2 $(p=.006)$.

Results. These results indicate that there are no significant differences in accuracy between all four techniques at breadth- 4 but that the two-handed simultaneous technique is less accurate than other techniques at breadth-8. 

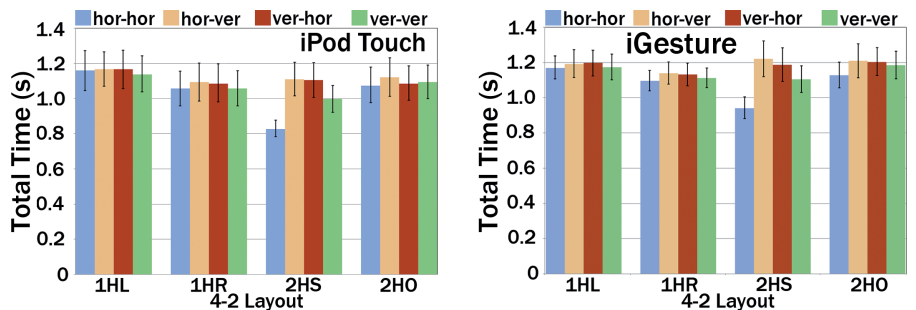

Fig. 9. Average total times for horizontal or vertical stroke groupings for the 4-2 layout using the iPod Touch (Left) and the iGesture (Right). 95\% confidence intervals are shown.
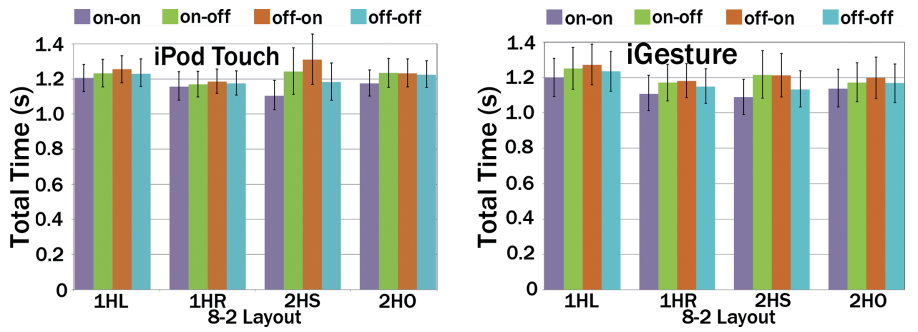

Fig. 10. Average total times for on- or off-axis stroke groupings for the 8-2 layout using the iPod Touch (left) and the iGesture (Right). 95\% confidence intervals are shown.

\subsection{4-2 Layout Stroke Groupings}

For the 4-2 layout, we compared horizontal-horizontal, horizontal-vertical, verticalhorizontal, and vertical-vertical groupings of stroke pairs. Total times for the stroke groupings are shown in Figure 9 . Conducting a 2 (device) $\times 4$ (technique) $\times 4$ (stroke grouping) repeated measures ANOVA with total time as the dependent variable, we found significant main effects for device $\left(F_{1,15}=9.24, p=.008\right)$, technique $\left(F_{3,45}=\right.$ $8.23, p<.001)$, and stroke grouping $\left(F_{3,45}=59.87, p<.001\right)$. On average, horizontalhorizontal strokes $(1057 \mathrm{~ms})$ strokes and vertical-vertical strokes $(1108 \mathrm{~ms})$ were faster than mixed stroke pairs (1157 ms for horizontal-vertical and $1146 \mathrm{~ms}$ for verticalhorizontal). We also found a significant two-way interaction between technique and stroke grouping $\left(F_{9,135}=35.44, p<.001\right)$; the 2 HS technique had a larger difference in speed between same-axis pairs and different-axis pairs, than the other techniques. In addition, for the $2 \mathrm{HS}$ condition, stroke pairs that were either bilaterally symmetric or in the same direction (translationally symmetric) $(894 \mathrm{~ms}$ ) were faster than the remaining pairs $(1147 \mathrm{~ms}$ ) by $28 \%$. For accuracy we found no significant main effect for stroke grouping nor any significant interactions between any of the factors.

Results. These results show that the average total times for same-axis pairs were faster than strokes along different axes and that the largest differences in speed occurred for the two-handed simultaneous technique.

\subsection{8-2 Layout Stroke Groupings}

We compared on-on, on-off, off-on, and off-off axis selections for the 8-2 layout. An on-axis stroke is one drawn in a cardinal direction while an off-axis stroke is one drawn along a diagonal. Total times for the stroke groupings are shown in Figure 10. Conducting a 2 (device) $\times 4$ (technique) $\times 4$ (stroke grouping) repeated measures ANOVA with total time as the dependent variable, we found significant main effects for technique $\left(F_{3,45}=3.90, p=.015\right)$ and stroke groupings $\left(F_{3,45}=46.59, p<.001\right)$. On average, on-on strokes (1148 ms) and off-off strokes (1188 ms) were faster than mixed stroke pairs (1212 ms for on-off and $1232 \mathrm{~ms}$ for off-on). There was also a significant 
two-way interaction between technique and stroke groupings $\left(F_{9,135}=6.01, p<.001\right)$, as the 2HS technique had a bigger speed difference between pairs that were on-on or off-off compared to the mixed pairs than the other techniques. We also found a significant three-way interaction $\left(F_{9,135}=2.73, p=.006\right)$. For accuracy, we found no significant main affect for stroke grouping nor any significant interactions between any of the factors.

Results. These results show that the average total times for on-on and off-off pairs were faster than mixed pairs and that the largest differences in speed occurred for the two-handed simultaneous technique.

\subsection{Temporal Overlap}

To assess the level of temporal overlap in the two-handed conditions, we computed the delay between the initial touch of the first hand and the initial touch with the second hand. A shorter delay indicates a greater likelihood of overlap between the hand motions. We performed a 2 (device) $\times 2$ (2HS and $2 \mathrm{HO}$ techniques) $\times 3$ (layout) repeated measures ANOVA with delay as the dependent measure. We found significant main effects for technique $\left(F_{1,15}=157.47, p<.001\right)$ and layout $\left(F_{2,30}=10.45, p<.001\right)$. The average delay was shorter for $2 \mathrm{HS}(44 \mathrm{~ms})$ than for $2 \mathrm{HO}(261 \mathrm{~ms})$. We also found an interaction between technique and device $\left(F_{1,15}=12.16, p=.003\right)$, as the delay for $2 \mathrm{HS}$ was slightly longer for the iGesture than the iPod Touch, but the delay for $2 \mathrm{HO}$ was slightly longer for the iPod Touch than for the iGesture.

Results. These results confirm that the two-handed simultaneous technique has a much shorter delay between initial touches and greater temporal overlap than the two-handed ordered technique.

\subsection{Starting Hand for Two-Handed Ordered}

For $2 \mathrm{HO}$ we compared whether using the left hand or the right hand for the first stroke had an impact on performance. We performed separate 2 (hand) $\times 3$ (layout) $\times 2$ (device) repeated measures ANOVAs on total time and accuracy. For total time, we found significant main effects for device $\left(F_{1,15}=6.65, p=.021\right)$ and layout $\left(F_{2,30}=\right.$ $229.12, p<.001)$. More interestingly, however, we found no significant main effect for hand $\left(F_{1,15}=0.31, p=.584\right)$, nor any interaction effects between any of the factors, which indicates that the starting hand does not significantly affect total time, regardless of layout and device. For accuracy, we found only a significant main effect for layout $\left(F_{2,30}=12.17, p<.001\right)$. Again, we found no significant main effect for hand $\left(F_{1,15}=2.10, p=.168\right)$, nor any significant interactions between hand and the other two factors, which indicates that there was no significant difference in accuracy due to starting hand, regardless of layout and device. The only significant two-way interaction was between device and layout $\left(F_{2,30}=4.97, p=.014\right)$.

Results. These results suggest that the starting hand does not affect performance (speed or accuracy) of the two-handed ordered technique.

\subsection{Single Stroke Direction}

To better understand which individual stroke directions are easiest to perform with the thumbs and fingers, we examined the movement time for the first stroke in the 8-2 layout for both of the one-handed conditions (Figures 11 and 12). One participant did not have any trials for one of the directions with the right hand in the iPod Touch condition, so we removed that person's data from the right hand analysis. After removing this data we could not perform a complete multi-way repeated measures ANOVA across all factors. Therefore, for the iPod Touch, we ran separate ANOVA's for each hand with stroke direction as the factor and we found that stroke direction had a significant effect on movement time for both the left hand $\left(F_{7,105}=3.74, p=.001\right)$ and the right hand $\left(F_{7,98}=3.46, p=.002\right)$. Examining the average movement times in more detail, we 


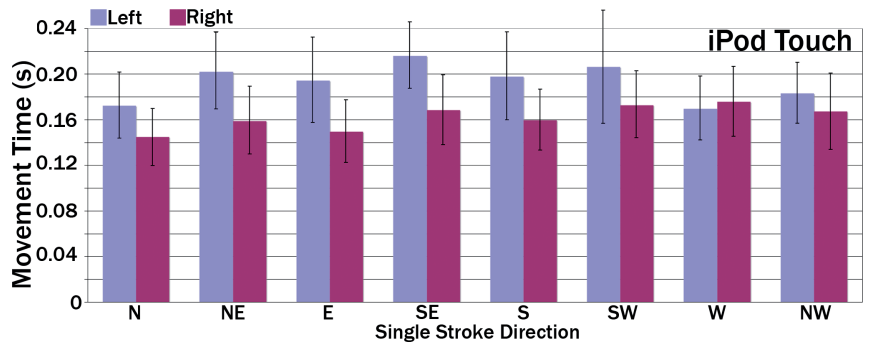

Fig. 11. First stroke average movement (with $95 \%$ confidence intervals) times using the iPod Touch for the 8-2 layout.

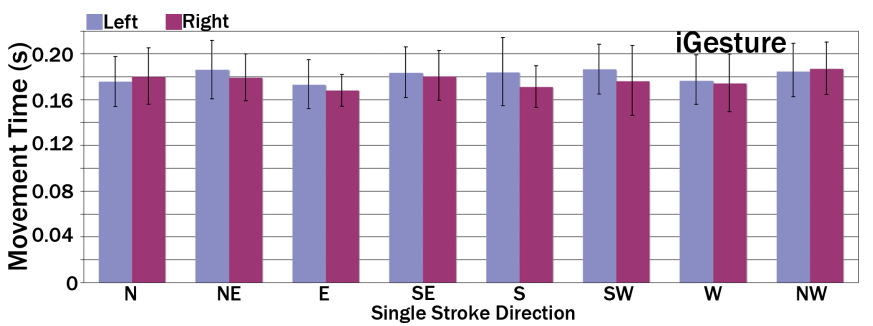

Fig. 12. First stroke average movement (with 95\% confidence intervals) times using the iGesture for the 8-2 layout.

found that the left thumb was fastest at selecting strokes in the upper left quadrant (N, NW, W directions) and the right thumb was fastest at selecting strokes in the upper right quadrant ( $\mathrm{N}, \mathrm{NE}, \mathrm{E}$ directions). In addition, on-axis stroke directions tended to be faster than their neighboring off-axis directions. Pooling the data across all directions, we found that the right thumb (161 ms) was significantly faster than the left thumb $(191 \mathrm{~ms})(p=.005)$ at drawing a single stroke.

Results. These results show that the right thumb is faster than the left thumb, and both thumbs are faster at moving upwards and inwards, with respect to the hands, than in other directions.

For the iGesture, we ran a two-way ANOVA comparing with hand and stroke direction as the factors. We found no significant main effects for hand $\left(F_{7,105}=0.83\right.$, $p=.565)$ or direction $\left(F_{1,15}=0.36, p=.559\right)$, and we found no significant interaction effect $\left(F_{7,105}=0.49, p=.840\right)$.

Results. These results indicate that on the iGesture, where a participant uses either the index or middle finger, neither the hand nor the direction significantly affects movement time of a single stroke.

\section{DISCUSSION}

The overall goal of our study was to investigate the performance of multistroke marking menus in the context of multitouch devices. We summarize the results as follows.

Two-Handed Simultaneous Menus Are as Fast as Dominant-Handed Multistroke Menus. We hypothesized (H1) that 2HS would outperform the other menu techniques because it maximizes temporal overlap in hand motion. However, the total time for $2 \mathrm{HS}$ was very similar to that for $1 \mathrm{HR}$ and we found no statistically significant differences between them. Although $2 \mathrm{HS}$ was fastest in movement time, 16.5-41.8\% faster on the iPod Touch and 19.4-36.4\% faster on the iGesture than 1HR, it also incurred a slower reaction time, indicating that users spent more time remembering and planning their strokes when coordinating simultaneous motions of two hands. These results do not allow us to accept hypothesis $H 1$. However, based on our own experience with $2 \mathrm{HS}$ 
we believe that with practice users can cognitively chunk the simultaneous two-stroke gestures and greatly reduce their reaction time. The two strokes proceduralize into a single automated action, like a form of "muscle memory." As described in the next section, we conducted a longitudinal study to test this hypothesis.

At breadth-4, 2HS was just as accurate as the other menu designs, but at breadth-8, which requires more precise motions and thus more careful coordination, $2 \mathrm{HS}$ was less accurate. Nevertheless accuracy remained above $82 \%$ across all conditions we tested and can improve significantly with practice as we will show in the next section.

Two-Handed Ordered Menus Are as Fast as Dominant-Handed Multistroke Menus and Provide Access to Twice as Many Items. Although we hypothesized (H2) that $2 \mathrm{HO}$ would be faster than the one-handed designs (1HR and 1HL) due to overlap in hand movement, we found that total time and accuracy were not significantly different between $2 \mathrm{HO}$ and $1 \mathrm{HR}$. The reaction times were faster for $2 \mathrm{HO}$ than for $2 \mathrm{HS}$, but slightly slower than for the one-handed menus. This result indicates that planning finger movements takes more time for the two-handed menus. Since starting with the right hand or left hand makes no significant difference on total time or accuracy for the $2 \mathrm{HO}$ design, both alternating orders are equally useful for selecting menu items.

Dominant-Handed Multistroke Marking Menus Are Faster than NondominantHanded Menus. Our results show, for one-handed marking menus, the right hand outperforms the left hand in total time for both the iPod Touch and iGesture conditions, confirming hypothesis H3. All of our subjects were right-handed, so we expected the right, dominant hand to be faster than the nondominant hand. Surprisingly, we found no significant difference in accuracy between the right and left hand marking menus, suggesting that the only penalty for using the nondominant hand is in speed.

Stroke Direction Affects Performance on the iPod Touch. When drawing a single stroke on the iPod Touch, we found a significant difference between hands, with the dominant hand faster than the nondominant hand. We also found that stroke direction had a significant effect on movement time. For each hand, participants were fastest at pulling their thumbs up or inward with respect to the hand, and on-axis strokes were faster than the neighboring off-axis strokes. For the slower stroke directions, the participants may have been limited by the rotational constraints of the thumbs while holding the iPod Touch, but when the participants could freely move their hands on the iGesture we found no significant difference between hands nor any significant differences due to stroke direction.

Some Stroke Pairs Are Faster to Draw than Others. We also examined how different groupings of stroke directions affect drawing speed. For the 4-2 layout, pairs of both vertical strokes and both horizontal strokes tended to have faster average total times than the mixed pairs. In the two-handed simultaneous condition, drawing bilaterally symmetric strokes or strokes in the same direction was faster than drawing nonmirrored strokes. For the 8-2 layout, on-on and off-off pairs were faster than the mixed pairs for the two-handed simultaneous design. On-on strokes were slightly faster than off-off strokes on the iPod Touch.

Two-Handed Simultaneous Exhibits More Temporal Overlap than Two-Handed Ordered. For the two-handed conditions, the delay between the start of the first stroke and the second stroke was significantly different between the simultaneous and ordered conditions by about $200 \mathrm{~ms}$. One direction for future work is to use this delay to distinguish which of these two menu techniques the user is performing.

\section{USER STUDY 2: LONGITUDINAL EVALUATION}

In our first study, we found that the significant improvement in movement time for the two-handed simultaneous technique was offset by an increase in reaction time required to process the stimulus and plan the hand movements. However, with enough practice, 

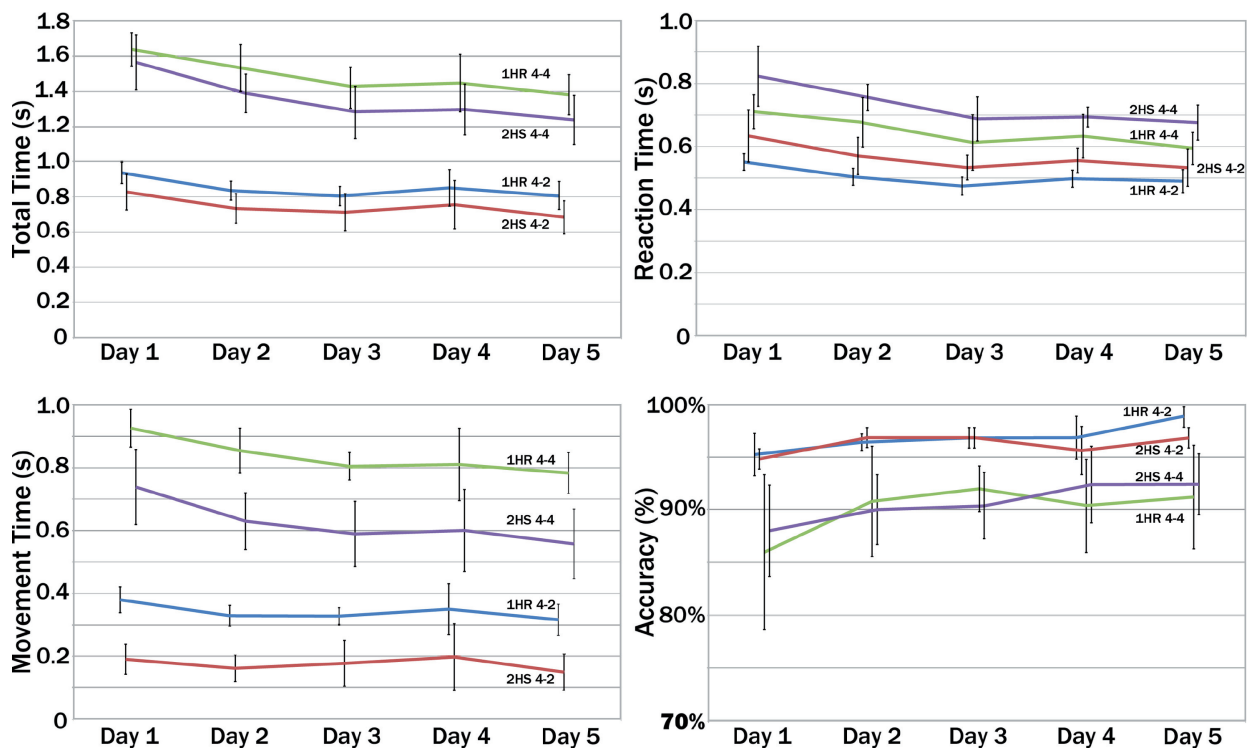

Fig. 13. Average time and accuracy (with 95\% confidence intervals) for menu techniques $1 \mathrm{HR}$ and $2 \mathrm{HS}$ and layouts 4-2 and 4-4, across five days. The baseline value of the accuracy graph is $70 \%$.

users may require less time to plan movements as drawing two strokes at the same time becomes more automatic. We hypothesize that with practice the following will occur.

H4. Reaction times for $1 \mathrm{HR}$ and $2 \mathrm{HS}$ will converge.

H5. The $2 \mathrm{HS}$ technique will outperform $1 \mathrm{HR}$ in total time.

To test these hypotheses, we conducted a longitudinal study with five right-handed participants ( 3 male, 2 female, between 24 and 32 years old) using the iPod Touch. We focused the longitudinal study on the iPod Touch as handheld multitouch devices are more ubiquitous than larger interactive surfaces like the iGesture. We gave each participant an iPod Touch to use over five consecutive days. Each participant spent approximately 45 minutes every day performing three blocks of 50 trials, using both the $1 \mathrm{HR}$ and $2 \mathrm{HS}$ menu techniques. The participants first performed the trials using the 4-2 layout and then the 4-4 layout. We used the same stimuli and feedback as in the first study. The participants received no practice trials and our analysis includes all data. As in the first study, our dependent variables were reaction time, movement time, total time, and accuracy. Misses were counted similarly, except the participants did not have to repeat missed sequences, as sequences were generated randomly.

\subsection{Longitudinal Results}

As shown in Figure 13, the total, reaction and movement times all decrease with practice while accuracy increases. Across both layouts 4-2 and 4-4, by day five, $2 \mathrm{HS}$ was $10-15 \%$ faster in total time and $29-52 \%$ faster in movement time than $1 \mathrm{HR}$. However, reaction time remained $10-12 \%$ slower for $2 \mathrm{HS}$ than for $1 \mathrm{HR}$, even after five days. Finally, on day five, accuracy was between $92.4-96.8 \%$ for $2 \mathrm{HS}$ and between 91.2-98.8\% for $1 \mathrm{HR}$.

For each layout (4-2, 4-4) and dependent variable (total time, movement time, reaction time, accuracy), we ran separate two-way repeated measures ANOVAs with day and menu technique (1HR, 2HS) as factors. We found significant main effects $(p<.05)$ in all but the following cases; (1) for menu layout $4-2$, day did not significantly affect 

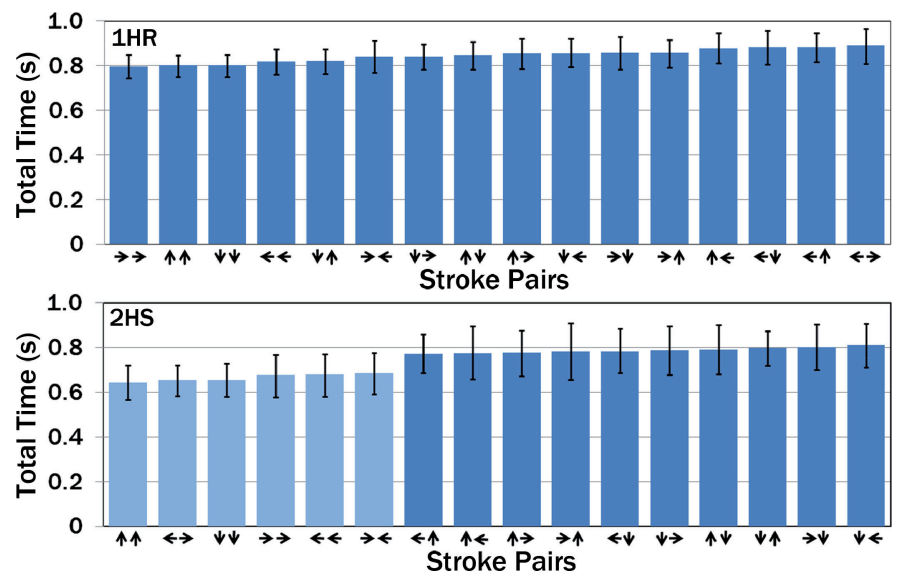

Fig. 14. Average total time (with 95\% confidence intervals) per stroke pair for the 4-2 layout. For 2HS, pairs of strokes that are bilaterally symmetric or share the same direction are $18 \%$ faster to draw than the other pairs.

movement time and menu technique did not significantly affect accuracy, (2) for menu layout 4-4, neither day nor menu technique significantly affected accuracy. We found no significant interactions in any of the ANOVAs. We include more detailed numerical results in the electronic appendix.

\subsection{Longitudinal Discussion}

Time and Accuracy. While we cannot accept our hypothesis (H4) that reaction times would converge, we found that the difference in reaction times between $1 \mathrm{HR}$ and $2 \mathrm{HS}$ did decrease significantly after five days. The decrease was $29.6 \%$ for the $4-2$ layout and $26.2 \%$ for the $4-4$ layout, suggesting that time required to coordinate two hand movements diminishes with practice. For the 4-2 layout, there was no significant difference in movement time across days. For the 4-4 layout, reaction and movement time improved for both techniques, but the movement time advantage for $2 \mathrm{HS}$ outweighed the reaction time advantage for $1 \mathrm{HR}$. Menu technique had a significant effect on total time, and by day five, total time was faster for $2 \mathrm{HS}$ than $1 \mathrm{HR}$ by $15.3 \%$ for layout $4-2$ and $10.3 \%$ for layout $4-4$, confirming hypothesis $H 5$. In our initial study, $2 \mathrm{HS}$ had a relatively low accuracy rate for the $4-4$ layout (89.5\%). Although we did not find the day to have a significant effect on accuracy, the average accuracy did improve from $88.0 \%$ to $92.4 \%$ after five days and there was no significant difference between the two techniques. Together, these results suggest that although $2 \mathrm{HS}$ may be more difficult to use than 1HR at first, with moderate practice an expert user can access menus items more quickly using $2 \mathrm{HS}$ than $1 \mathrm{HR}$, while maintaining reasonably good accuracy.

Stroke Directions. Figure 14 shows the total time in sorted order for each of the 16 stroke pairs in the 1HR condition (top) and the $2 \mathrm{HS}$ condition (bottom). For $2 \mathrm{HS}$, we find that drawing strokes that are bilaterally symmetric or in the same direction (translationally symmetric), is 18\% faster on average than drawing the remaining stroke pairs. For $1 \mathrm{HR}$, we find no large difference in total time between groups of stroke pairs, but the pairs in which both strokes are drawn in the same direction are the four fastest.

Our $2 \mathrm{HS}$ results indicate that users are most efficient at drawing symmetric stokes and are consistent with prior studies of two-handed motion control [Kelso 1984; Mechsner et al. 2001]. Together, these studies suggest that people find it more difficult to draw pairs of asymmetric strokes than symmetric strokes. However, the stimuli for 

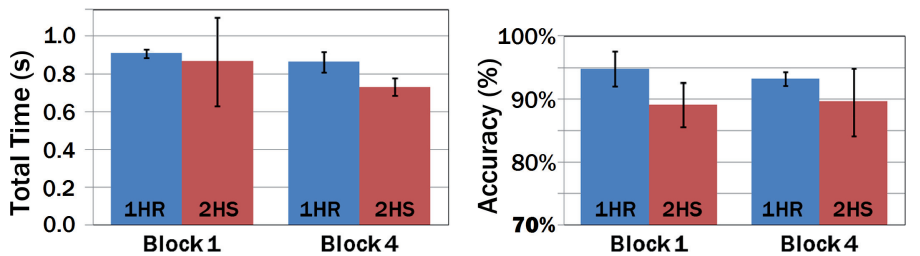

Fig. 15. Average total time and accuracy (with $95 \%$ confidence intervals) for the first and last blocks for the 8-2 layout. The baseline value of the accuracy graph is $70 \%$.

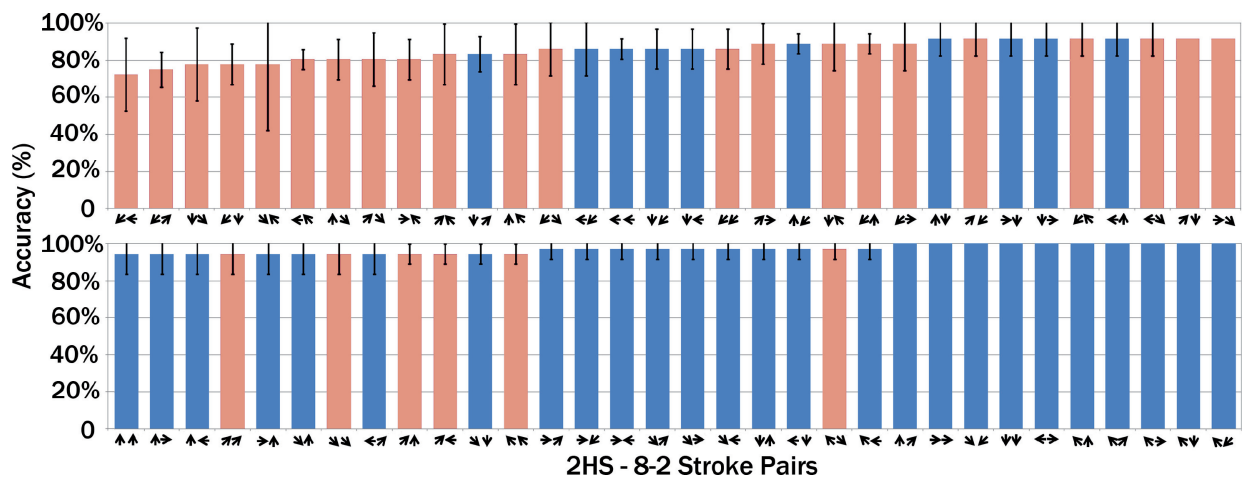

Fig. 16. Average accuracy (with 95\% confidence intervals) for all stroke pairs in the 8-2 layout using the $2 \mathrm{HS}$ technique. The pairs where the left stroke is parallel to the SW-NE axis or the right stroke is parallel to the SE-NW axis are highlighted in orange. Note that confidence intervals have zero radius when all three participants had the same accuracy.

the task may also affect performance. Balakrishnan and Hinckley [2000] and Franz et al. [2001] have shown that users perform two-handed tasks more efficiently when the stimuli are seen as representing a single task rather than two independent tasks. We leave it to future work to investigate how the visual design of the stimuli might affect performance.

\subsection{Longitudinal Study: 8-2 Menu Layout}

Based on the results of our longitudinal study on the breadth-4 menus we conducted a small follow up experiment with three right-handed participants ( 2 male, 1 female, between 24 and 25 years old) to examine long term performance for the breadth-8 layout using menu techniques $2 \mathrm{HS}$ and $1 \mathrm{HR}$. Each participants performed four blocks of trials, where each block consisted of three repetitions of the 64 stroke pairs in randomized order for each menu technique. Again, the participants received no practice trials and our analysis includes all data.

As shown in Figure 15, by the fourth block the total time was $15.4 \%$ faster with $2 \mathrm{HS}$ than with $1 \mathrm{HR}$ and average accuracies were $89.6 \%$ for $2 \mathrm{HS}$ and $93.2 \%$ for $1 \mathrm{HR}$. The speedup was in line with our breadth-4 results. Moreover, the $2 \mathrm{HS}$ accuracy of $89.6 \%$ was much higher than the $82.3 \%$ we saw in our initial study of the 8-2 layout, suggesting that practice can improve accuracy. Although $2 \mathrm{HS}$ was not quite as accurate as $1 \mathrm{HR}$, examining the individual stroke pairs for $2 \mathrm{HS}$ (Figure 16), we found that when the left stroke was parallel to the SW-NE axis or the right stroke was parallel to the SE-NW axis accuracy (highlighted in orange) dropped to $86.6 \%$, while the remaining 36 stroke pairs maintained an accuracy at 95.0\%. Our results confirm Karlson et al.'s [2005] observation that for the right thumb, SE-NW strokes are the most difficult 


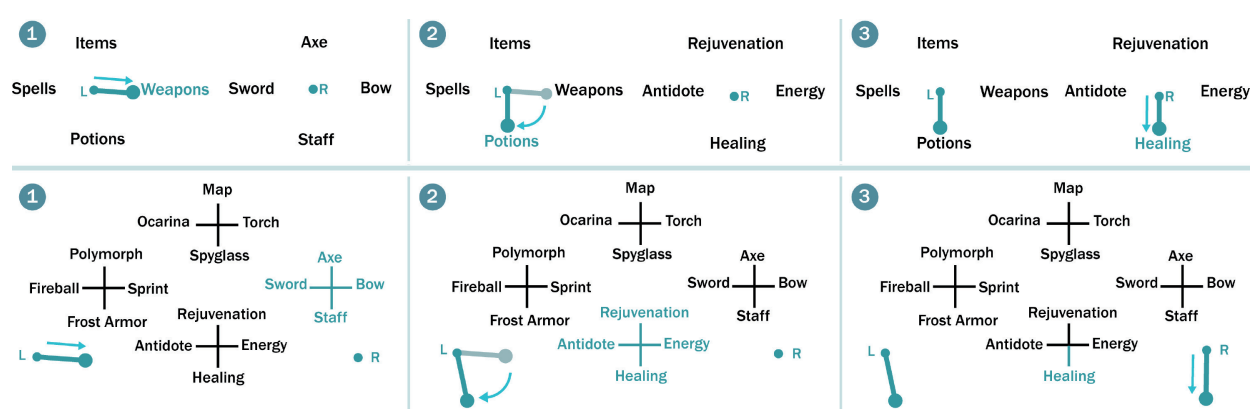

Fig. 17. Top: Hierarchical Display_-The left hand explores the parent menu items by dragging through menu items, and the child menu items continuously update for the right hand. Bottom: Full-Breadth Display-The entire menu space is displayed, and the left hand chooses the four-item cluster, while the right hand chooses the item within a cluster.

to draw. Just as for the breadth-4 layout, we found that pairs of bilaterally symmetric or same-direction strokes $(731 \mathrm{~ms})$ were faster to draw than other pairs $(815 \mathrm{~ms})$.

\section{DESIGN GUIDELINES}

Based on our results we make the following design recommendations. Two-handed simultaneous multistroke marking menus provide the fastest performance with good accuracy at breadth- 4 and acceptable accuracy at breadth- 8 . The most frequently used commands should be bound to pairs of bilaterally symmetric strokes or same-direction strokes as they are the fastest to perform. For handheld thumb-operated devices it may be possible to improve breadth- 8 accuracy by avoiding the use of the SW-NE directions for the left thumb and the SE-NW directions for the right thumb. Alternatively, designers may bind commands that require conscious commitment, such as deletion or quitting, to these pairs that are harder to perform.

With a one-handed marking menu operated by a thumb, the right thumb is faster than the left thumb. Within the right-handed menu the most frequently used menu items should be placed in the upper right quadrant of directions, and within the lefthanded menu, the most frequently used menu items should be placed in the upper left quadrant, as those directions had the fastest movement times.

\section{DISPLAYING MENU ITEMS FOR NOVICE USERS}

Although our studies focused on expert performance, real-world usage of our twohanded designs requires methods for training novice users in the mapping between stroke pairs and menu items. We offer two novice-mode visualizations that facilitate exploration of the menu items bound to stroke pairs.

In a hierarchical display (Figure 17 Top), the left hand selects the parent menu item by dialing through the items [Zhao et al. 2007]. The right menu continuously updates to show the corresponding child menu items. The closest parent and child menu items are always highlighted to indicate which option the user has currently chosen. Users can continuously explore all possible menu items without backtracking or lifting up any fingers. However, navigating a four-stroke menu would still require backtracking if the wrong initial pair was selected.

In a full-breadth display (Figure 17 Bottom), all the items are presented [Zhao et al. 2006], and the left hand specifies the menu cluster, while the right hand specifies the item within a cluster. The cluster that the left hand is currently selecting is highlighted as feedback to the user. The user can dynamically switch to any of the options without lifting up any fingers. This display can be particularly useful when there is no logical way to group items into equally sized clusters. 

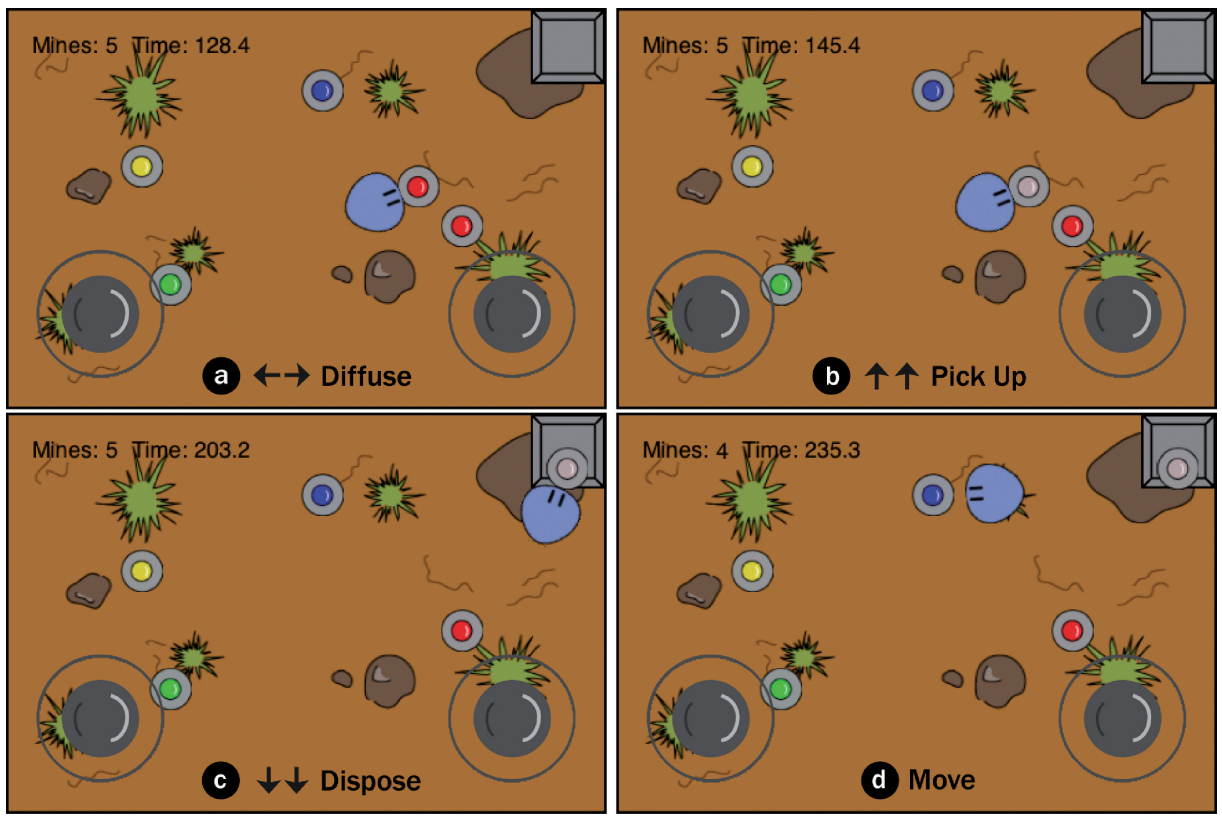

Fig. 18. In this mine disposal game, the user moves a robot using two joysticks. The left joystick controls movement and the right joystick controls orientation. Two-handed marking menus invoke commands as shown in the four screenshots and can be executed anywhere on the screen.

\section{APPLICATIONS}

We have shown that our two-handed multistroke marking menus are viable menu selection techniques. Although many iPhone/iPod Touch applications and games use two-thumb controls we have not yet seen two-thumb marking menus on these devices. We believe many such handheld applications could benefit from our two-thumb marking menus and we present several usage scenarios.

Dual-Joystick Navigation. Many iPhone games require players to hold the device in landscape mode and use two virtual joysticks operated by the thumbs, one to control movement and the other to control character orientation. Our two-thumb techniques could be integrated into such games and used to trigger commands such as diffusing or disposing of mines in a mine disposal game (Figure 18). For example, we could integrate our marking menus by overlaying them on the joysticks. In this case users would need to use some distinguishing gesture to indicate whether they wished to invoke the marking menus or use the joysticks. For instance quick strokes might indicate menu selection while longer strokes might indicate joystick-based movements. Another option would be to interpret all strokes that lie outside the joystick regions as menu commands. However, this approach might require users to repeatedly move their hands on and off the joystick regions. We utilize the first approach, and interpret strokes under 0.25 seconds in duration as menu commands. Because our techniques are eyes-free, expert users can keep their focus on the current task and select commands without visually searching for soft buttons to select a command. Examining data from a small, informal sample of two members of our lab we have found that expert users can switch from joystick-based movement to two-handed menu selection and back to movement in less than 0.5 seconds.

Text Editing. In text-editing mode on touchscreen devices such as the iPhone, the keyboard takes up half the screen, leaving little room for text formatting options. 


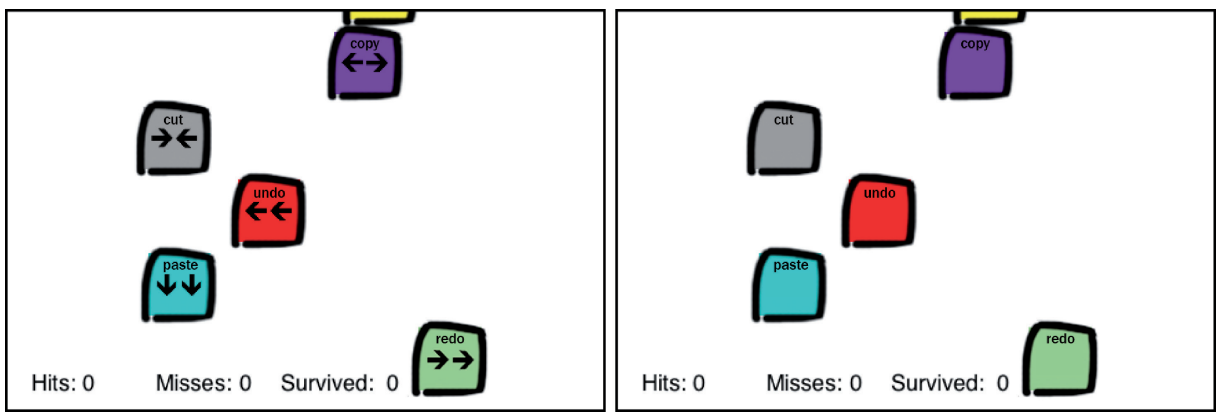

Fig. 19. In the Falling Blocks game, the user must destroy each block by drawing the corresponding strokes. Left: Novice Mode-Strokes are shown. Right: Expert Mode-No strokes are shown.

We have integrated our two-handed marking menus into a text-editing application allowing users to quickly change the attributes of the text (Figure 1) by selecting from a two-handed marking menu, located above the keyboard, rather than through soft buttons.

Falling Blocks Game for Training Novice Users. Touch-typing is a complex task that requires coordination of ten fingers. Novice typists often use typing games to become proficient. Similarly, we offer a Falling Blocks game to train novices to quickly draw two directional strokes simultaneously to execute commonly used system commands. In the game, colored blocks continually fall down the screen, and each block is associated with a command and a stroke pair. Users must execute the correct strokes to destroy each block before it falls to the bottom of the screen. In novice mode, the strokes are shown to the user, but in expert mode, the user must remember the mapping between commands and strokes (Figure 19).

The iPhone App Store provides an opportunity for researchers to introduce applications to the general public. As an experiment, we released Block Blender, a variant of our Falling Blocks application, on the App Store. In the game, blocks continuously fall down the screen and the player must draw the specified pair of strokes to destroy the block. When the stack of blocks reaches the top of the screen, the game is over. We had nearly 1000 downloads after the first seven weeks of deployment. Users could opt to send scores and data from their completed games back to our servers. Of the scores we received, two players destroyed at least 1000 blocks with $93.7 \%$ and $85.1 \%$ accuracy. Six players were able to outscore the first author's best score of 184, each doing so in six tries or less. These players were able to stay alive with a new block generated every 0.82 seconds while performing pairs of strokes with average movement times between 0.13 second and 0.29 seconds. Although this application demonstrates that some players can quickly pick up the skill to draw pairs of strokes simultaneously and outperform even the first author, most players did not produce such high scores. These players may still be learning the mechanics of the game.

\section{CONCLUSION}

Two-handed multistroke marking menus are effective menu selection techniques for multitouch devices. Our studies of expert-level performance show that the simultaneous variant is faster than the one-handed designs and performs with reasonable accuracy. The ordered variant doubles the number of accessible menu items. Multitouch devices from handheld devices to large-scale tables and walls are rapidly becoming mainstream technologies. We believe that techniques like two-handed marking menus are a step towards exploiting the full potential of these devices. 


\section{ELECTRONIC APPENDIX}

The electronic appendix for this article can be accessed in the ACM Digital Library

\section{ACKNOWLEDGMENT}

We would like to thank Tony DeRose for his invaluable input.

\section{REFERENCES}

APPLE. Apple iPhone. http://www.apple.com/iphone/.

Bailly, G., Lecolinet, E., AND NigaY, L. 2008. Flower menus: A new type of marking menu with large menu breadth, within groups and efficient expert mode memorization. Proc. AVI, 15-22.

BALAKRIShNAN, R. AND Hinckley, K. 1999. The role of kinesthetic reference frames in two-handed input performance. In Proceedings of the ACM Symposium on User Interface Software and Technology (UIST). 171-178.

Balakrishnan, R. ANd Hinckley, K. 2000. Symmetric bimanual interaction. In Proceedings of the SIGCHI Conference on Human Factors in Computing Systems (CHI). 33-40.

Balakrishnan, R. and Patel, P. 1998. The PadMouse: Facilitating selection and spatial positioning for the non-dominant hand. In Proceedings of the SIGCHI Conference on Human Factors in Computing Systems (CHI). 9-16.

Buxton, W. 1986. Chunking and phrasing and the design of human-computer dialogues. In Proceedings of the IFIP World Computer Congress, 475-480.

Buxton, W. And Myers, B. 1986. A study in two-handed input. In Proceedings of the SIGCHI Conference on Human Factors in Computing Systems (CHI). 321-326.

Casalta, D., Guiard, Y., and Beaudouin-Lafon, M. 1999. Evaluating two-handed input techniques: Rectangle editing and navigation. In Proceedings of the SIGCHI Conference on Human Factors in Computing Systems (CHI) Extended Abstracts. 236-237.

FINGERWORKS. iGesture. http://www.fingerworks.com.

Franz, E., Zelaznik, H., Swinnen, S., and Walter, C. 2001. Spatial conceptual influences on the coordination of bimanual actions: When a dual task becomes a single task. J. Motor Behav. 33, 1, 103-112.

GuIARD, Y. 1987. Asymmetric division of labor in human skilled bimanual action: The kinematic chain as a model. J. Motor Behav. 19, 4, 486-517.

Hinckley, K., Pausch, R., Proffitt, D., Patten, J., and Kassell, N. 1997. Cooperative bimanual action. In Proceedings of the SIGCHI Conference on Human Factors in Computing Systems (CHI). 27-34.

Isokoski, P. AND KäKI, M. 2002. Comparison of two touchpad-based methods for numeric entry. In Proceedings of the SIGCHI Conference on Human Factors in Computing Systems (CHI). 25-32.

Kabbash, P., Buxton, W., And Sellen, A. 1994. Two-handed input in a compound task. In Proceedings of the SIGCHI Conference on Human Factors in Computing Systems (CHI). 417-423.

Karlson, A. K., Bederson, B. B., and SanGiovanni, J. 2005. AppLens and LaunchTile: Two designs for onehanded thumb use on small devices. In Proceedings of the SIGCHI Conference on Human Factors in Computing Systems (CHI). 201-210.

Kelso, J. 1984. Phase transitions and critical behavior in human bimanual coordination. Amer. J. Phys. 15, R1000-R1004.

KöLtringer, T., IsoKoski, P., AND GREChenIG, T. 2007. TwoStick: Writing with a game controller. In Proceedings of the Symposium on Graphics Interface, 103-108.

Kuntenbach, G. 1993. The design and evaluation of marking menus. Ph.D. dissertation, University of Toronto.

Kurtenbach, G. AND Buxton, W. 1993. The limits of expert performance using hierarchic marking menus. In Proceedings of the SIGCHI Conference on Human Factors in Computing Systems (CHI). 258-264.

Kuntenbach, G. AND Buxton, W. 1994. User learning and performance with marking menus. In Proceedings of the SIGCHI Conference on Human Factors in Computing Systems (CHI). 258-264.

Latulipe, C., Kaplan, C., AND Clarke, C. 2005. Bimanual and unimanual image alignment: An evaluation of mouse-based techniques. In Proceedings of the ACM Symposium on user Interface Software and Technology (UIST). 123-131.

Latulipe, C., Mann, S., Kaplan, C., and Clarke, C. 2006. SymSpline: Symmetric two-handed spline manipulation. In Proceedings of the SIGCHI Conference on Human Factors in Computing Systems (CHI). $349-358$. 
Lepinski, G. J., Grossman, T., and Fitzmaurice, G. 2010. The design and evaluation of multitouch marking menus. In Proceedings of the SIGCHI Conference on Human Factors in Computing Systems (CHI). 2233-2242.

McGuffin, M. J., Burtnyk, N., ANd KuRtenbach, G. 2002. FaST Sliders: Integrating marking menus and the adjustment of continuous values. In Proceedings of the Symposium on Graphics Interface, 35-41.

Mechsner, F., Kerzel, D., Knoblich, G., And Prinz, W. 2001. Perceptual basis of bimanual coordination. Nature 414, 69-73.

MERL. MERL DiamondTouch. http://merl.com/projects/DiamondTouch/.

Microsoft. Microsoft Surface. http://www.microsoft.com/surface.

Moyle, M. And CockBurn, A. 2002. Analysing mouse and pen flick gestures. In Proceedings of the SIGCHI Conference on Human Factors in Computing Systems (CHI). 19-24.

Odell, D. L., Davis, R. C., Smith, A., ANd Wright, P. K. 2004. Toolglasses, marking menus, and hotkeys: A comparison of one and two-handed command selection techniques. In Proceedings of the Symposium on Graphics Interface, 17-24.

Owen, R., Kurtenbach, G., Fitzmaurice, G., Baudel, T., and Buxton, W. 2005. When it gets more difficult, use both hands: Exploring bimanual curve manipulation. In Proceedings of the Symposium on Graphics Interface, 17-24.

PerceptivePixel. Perceptive Pixel. http://www.perceptivepixel.com/.

PerLIN, K. 1998. Quikwriting: Continuous stylus-based text entry. In Proceedings of the ACM Symposium on user Interface Software and Technology (UIST). 215-217.

Potter, R. L., Weldon, L. J., ANd ShNeiderman, B. 1988. Improving the accuracy of touch screens: an experimental evaluation of three strategies. In Proceedings of the SIGCHI Conference on Human Factors in Computing Systems (CHI). 27-32.

WEEGIE. http://weegie.sourceforge.net.

Wilson, A. D. and Agrawala, M. 2006. Text entry using a dual joystick game controller. In Proceedings of the SIGCHI Conference on Human Factors in Computing Systems (CHI). 475-478.

Хвох. http://www.xbox.com/.

Yatani, K., Partridge, K., Bern, M., and Newman, M. W. 2008. Escape: A target selection technique using visually-cued gestures. In Proceedings of the SIGCHI Conference on Human Factors in Computing Systems (CHI). 285-294.

Zhao, S., Agrawala, M., AND Hinckley, K. 2006. Zone and polygon menus: Using relative position to increase the breadth of multi-stroke marking menus. In Proceedings of the SIGCHI Conference on Human Factors in Computing Systems (CHI). 1077-1086.

ZhaO, S. ANd BalakRishnan, R. 2004. Simple vs. compound mark hierarchical marking menus. In Proceedings of the ACM Symposium on User Interface Software and Technology (UIST). 33-44.

Zhao, S., Dragicevic, P., Chignell, M., Balakrishnan, R., and Baudisch, P. 2007. Earpod: Eyes-free menu selection using touch input and reactive audio feedback. In Proceedings of the SIGCHI Conference on Human Factors in Computing Systems (CHI). 1395-1404.

Received June 2010; revised November 2010; accepted March 2011 